\title{
Pediatric Dentistry: Challenges during COVID-19 Pandemic
}

\author{
Sanhati Biswas ${ }^{1}$, Puloma Bagchi ${ }^{1}$, Nilotpol Kashyap ${ }^{2 *}$ \\ ${ }^{1}$ Post Graduate Student, Department of Pediatric and Preventive Dentistry, Rungta College of Dental Sciences \\ and Research, Bhilai, India \\ ${ }^{2}$ Professor, Department of Pediatric and Preventive Dentistry, Rungta College of Dental Sciences and Research, \\ Bhilai, India \\ *Corresponding Author: Nilotpol Kashyap, Professor, Department of Pediatric and Preventive Dentistry, \\ Rungta College of Dental Sciences and Research, Bhilai, India; Email: nilkash9365@gmail.com
}

Received Date: 10-09-2021; Accepted Date: 31-09-2021; Published Date: 07-10-2021

Copyright $^{\odot} 2021$ by Kashyap N, et al. All rights reserved. This is an open access article distributed under the terms of the Creative Commons Attribution License, which permits unrestricted use, distribution and reproduction in any medium, provided the original author and source are credited.

\begin{abstract}
In the present scenario when the whole world is fighting against the deadly COVID-19 virus, oral health of children is of great concern for the pediatric dentists. Children are now unwillingly locked inside their home and as a result they are in continuous habit of frequent food intake which is giving rise to various oral health issues. Various researches have also suggested children may play role as asymptomatic carrier of the infection and may lead to further spread of infection, but mortality rate is very negligible. So it is important to provide preventive measures for children for better oral health with minimal intervention and by remote information and education of caregivers and by using teledentistry. Moreover it is of utmost importance to follow the protective protocols while treating the child patients in the dental clinic to prevent cross-infection. This review is aimed at providing information regarding management of pediatric dental patients in midst of ongoing pandemic by following necessary protocols and preventive measures to prevent spread of COVID-19.
\end{abstract}

\section{Keywords}

Pandemic; Oral Health; COVID-19 Virus; Infection 


\section{Introduction}

The ongoing pandemic of COVID-19 is a challenge for the whole world including the scientific and medical society. Severe Acute Respiratory Syndrome Coronavirus 2 (SARS-CoV-2) is a complex disease with unpredictable clinical course. It develops rapidly and cause severe and fatal complications [1]. (SARSCoV-2), the seventh human coronavirus, was first detected in Wuhan, Hubei province, China, during the recent epidemic of pneumonia in January 2020 [2]. Since then, the virus has spread all over the world, and as of 20 May 2020, it has infected 4,806,299 people, and caused 318,599 deaths. The virus SARS-CoV-2 as well as SARS-CoV and Middle East respiratory syndrome coronavirus (MERS-CoV) cause severe pneumonia with a mortality rate of $2.9 \%, 9.6 \%$ and $36 \%$, respectively. The other four human coronaviruses, OC43, NL63, HKU1 and 229E, generally cause self-limited disease with mild symptoms. Other symptoms, such as gastrointestinal symptoms, and severe outcomes like stroke, blood coagulation disorders and possibly hyper-inflammation, have also been seen in many cases. Children mostly don't show severe symptoms unlike adults and death among children is negligible, but children in many countries have been subjected to the same social isolation like adults. Children aged less than 10 years account for $1 \%$ of COVID-19 according to China's Centres for Disease Control and Prevention. Out of 2,336,615 laboratory confirmed cases reported to the United States of America Centres for Disease Control and Prevention (CDC), only $4.5 \%$ were in children till June 24, 2020 [3]. There are various studies going on to determine the role children play in transmitting the virus that causes COVID-19. Transmission by children have quickly become an issue as school going children have to report to schools as soon as the imposed lockdown ends and whether it will be safe for the children to join the schools at this point of time is of great concern. There is also an increased risk of COVID-19 transmission due to gathering after opening of schools.

Maintaining the oral health of children becomes crucial during the COVID-19 pandemic by implementing specific protocols and preventive measures relating both to the pathologies of the oral cavity that normally exclude an emergency and to those clinical situations within the category of pediatric dental emergencies. For both the clinical situations, our main aim is to limit the spread of the disease and the onset of cross-infections. It calls for not only meticulous but also highly effective infection control protocols in the dental environments of the areas affected by COVID-19. It is also essential to progress on remote communication and education for better maintenance of oral health of children.

\section{COVID-19 Virus and Its Pathogenesis}

Coronavirus is an enveloped plus-strand RNA virus that belongs to the order family Coronaviridae and is classified into 4 primary genera: 
1. Alpha-coronavirus

2. Beta-coronavirus

3. Gamma-coronavirus

4. Delta-coronavirus

Mammals and humans are mostly affected by the $\alpha$ and $\beta$ variants. The $\gamma$ and $\delta$ genera infect birds. The bat is considered as one of the main reservoirs of the coronavirus according to various studies.

The main mechanism for SARS-CoV-2 infection is the binding of the virus to membranebound form of Angiotensin-Converting Enzyme 2 (ACE2) and the internalization of complex by the host cell. ACE2, a glycoprotein and metalloprotease, exists in both membrane-bound and soluble forms. The membrane-bound form contains a transmembrane domain which anchors its extracellular domain to the plasma membrane, whereas in its soluble form, it is cleaved and secreted, as the N-terminal ectodomain is barely measurable in circulation [4]. SARS-CoV-2 virus binds to a human cell receptor that expresses Angiotensin-Converting Enzyme 2 (ACE2). After binding there is a series of processes which includes internalization, replication, and the release of new virions from the infected cell. The coronavirus comprises of mainly 4 structural proteins: Spike (S), Membrane (M), Envelope (E) and Nucleocapsid (N). The spike protein is responsible for binding to ACE2 and causes in membrane fusion via conformational changes in the cell membrane. This process affects target organs such as lungs, digestive tract, heart, blood vessels, and kidneys where ACE2 expression is very high. This in turn leads to local and systemic inflammatory responses involving the affected organ.

\section{Characteristics of COVID-19 in Children}

According to recent literatures, in children fever and cough are the most usual signs of COVID19 after SARS-CoV-2 infection. Many studies conclusively confirmed the clinical impression that COVID-19 in children typically presents as a mild (37\%) or moderate $(45 \%)$ upper respiratory tract infection and is rarely severe or critical.

Viral shedding in asymptomatic cases was found in children aged 0-14 years and were considered as asymptomatic carriers and these asymptomatic carriers had lower peak of immunoglobulin M (IgM) against COVID-19, but they have shorter Ribonucleic Acid (RNA) negative conversion. Zhang, et al., compared conversion of 12 days versus 16 days in presymptomatic and symptomatic COVID-19 patients [5].

Luo, et al., reviewed 4950 close contacts and examined them according to mode of contact and clinical characteristics. An unpublished study from Guangzhou, China discovered strong 
association between disease severity and COVID-19 transmission $(\mathrm{P}<.0001)$. The symptoms which are associated with an elevated risk of disease transmission were fever, but not cough, fatigue or myalgia. Viral transmission can also take place when individuals touch their eyes, nose and throat, if the hand contains viral particles. Presently kindergartens and other primary and secondary educational institutions are implementing and promoting hygienic hand washing to counteract that risk.

Another study showed, 8 of 10 children were detected positive by Reverse Transcription-PCR (RT-PCR) on rectal swabs even though their nasopharyngeal testing was negative. It still need much research to find out whether the faecal positivity by RT-PCR represents residual viral genomic material or an active viral replication which is capable of causing infection. The presence of viral nucleo-capsid protein in gastric, duodenal, and rectal epithelia after isolation of SARS-CoV-2 from faecal samples, indicate that the virus actively infects these gastrointestinal glandular epithelial cells which means faecal-oral route of transmission is also possible [6].

\section{COVID -19 Risk Factors with Pediatric Patients}

In a dental operatory, oral fluids from the patient or contaminated dental instruments or environmental surfaces is one of the major way of spreading the virus to the dental surgeon, dental assistant and to other patients. The first gateway of infection is mostly by direct transmission through the respiratory droplets. The virus can get transmitted between the dentist, the dental assistant and the pediatric patients through the oral and nasal droplets released by coughing and sneezing or by the dental procedure itself. The suspended droplets containing the virus settle on the surfaces of the instruments with consequent transmission when the pediatric dentist, the assistant in the chair and other patients come into contact with the contaminated surfaces an indirect transmission can occur. There is extended risks of transmission by pediatric patients due to the use of removable orthodontic appliances or elements in fixed orthodontic therapies like inter-maxillary elastic bands, which increases risks of contamination if handling is not done with all precautions. Other problem which is most commonly encountered is difficulty for the child to use or manage Personal Protective Equipment (PPE) during their medical/dental visits. Last but not the least very presence of parents or attendants, with whom the pediatric dentist have to interface, also increases the risk of spread of infection.

\section{Coronavirus Disease 2019 (COVID-19) Pandemic: Interim Infection Prevention and Control Guidance for Dental Settings}

According to Centers of Disesse control and prevention interim guidance is updated based on currently available information about COVID-19 and the current scenario. Dentistry includes

Biswas S | Volume 2; Issue 2 (2021) | JDHOR-2(2)-032 | Short Communication

Citation: Kashyap N, et al. Pediatric Dentistry: Challenges during COVID-19 Pandemic. J Dental Health Oral Res. 2021;2(2):1-9. 
use of rotary dental and surgical instruments, such as air-rotor handpieces or ultrasonic scalers and air-water syringes. Surgical masks gives protection to mucous membranes of the mouth and nose from droplets suspended in the surrounding environment, but they fail to provide complete protection against inhalation of infectious agents (Fig. 1) [6].

- Implement Teledentistry

- It is important to telephone screen dental patient before their arrival in the dental clinic. Patient informing about any symptom associated with COVID-19 minor dental care should be avoided and delayed until the patient has ended isolation or quarantine

o The dentist need to assess the patient's dental condition and decide whether it is essential for the patient to be seen in the dental setting or it can be solved over teledentistry

- It should be advised to the patients and the person accompanying them to the appointment, to wear a cloth face covering or facemask when entering the clinic and that they will undergo thermal screening for fever and symptoms similar to COVID-19

- Temperature check: Any patient with elevated body temperature should avoid visiting clinic assuming them as potential carriers of virus. Infra-red forehead thermometer can be used to check the body temperature of the patient and self-declaration form should be signed by the patient or by the parents accompanying the pediatric patients prior to the dental procedure [7]

- Hand hygiene: Hand hygiene is must before and after patient examination, dental procedures. Also after coming in contact with the surroundings and equipment and after contacting the mucosa, damaged wound, blood, or any body fluid and secretion

- Precautionary measures for patient attenders: While attending pediatric patients, presence of parents or attenders is unavoidable. In that case attender should be minimized to a single parent. Since, the attender will be in the operational area they are also a potential threat as carriers of disease. If they are allowed inside the dental operatory, the attenders should be advised not to touch any of the equipment and operatory surfaces with bare hands. To reduce the anxiety of the child during dental procedure, a transparent screen/barrier can be made in the operatory between the dental chair and the attender area, where the attender can be present to observe the procedure

- Personal protective measures: A Personal Protective Equipment (PPE) should be worn on the outside over the autoclavable scrubs. Three-layered face protection which includes an N95 respirator, a surgical mask, and then a face shield is recommended for additional protection. Along with this, disposable head cap, disposable latex/nitrile gloves, shoe covers, protective goggles should also to be used for additional protection

- Pre-operational antimicrobial mouth rinse: Many studies have suggested that chlorhexidine ineffective against COVID-19. Use of oxidative agents such as $1 \%$ hydrogen peroxide or 
$0.2 \%$ povidone can be used as anti-microbial pre-operational mouthwash as suggested by National Health Commission of the People's Republic of China

- Aerosol-generating procedures: Dental equipments like ultrasonic and sonic scalers, air polishing, air-water syringe, high-speed handpieces and slow-speed handpieces produce aerosols. In such cases, high-power suction and dental dams is recommended to reduce spatter of droplets and aerosols. In cases where high-speed handpieces and dental ultrasonic devices are used rubber dams reduces the generation of contaminated airborne particles. Ventilation of the operational room is another concern. A well ventilated room will facilitate the escape of the generated aerosols and droplets and reduce the chances of contamination

- Sterilization and disinfection: Efficacious infection-control procedures should be observed by all dental surgeons and other dental operators. The exterior of the dental handpiece can be sterilised by autoclaving and interior can be sterilized by chemiclave

- Radiographic examination: Extra-oral radiographs should be considered over intraoral radiographs wherever possible. It is better to avoid intraoral techniques as may produce coughing. During an intraoral radiograph, sensor/film should be covered with an additional disposable plastic sleeve that is changed for every single patient

- Medical waste disposal: Marking of all medical waste, especially personal protective materials is important before disposal. They should be transported regularly to the depository area, and must be disposed of following the required waste management protocol [8]

- Maxillofacial injury: In any incidence of severe oral and maxillofacial trauma, like fracture of the maxillo-facial complex the patient should be hospitalized. A chest CT should be prescribed to exclude suspected infection as RT-PCR tests are time consuming and require specific labs

\section{Preventive Guidelines}

In present situation children are now unwillingly locked inside their home and as a result they are in continuous habit of frequent food intake which is giving rise to various oral health issues. At this time minimum dental check-ups and optional procedures are being performed, it has increased dental disease load. To keep this in check, certain preventive guidelines can easily be followed at home. Parents should be advised to:

- Not to place a child to sleep with a bottle in mouth

- Avoid using pacifiers dipped in sugar-containing food like honey, sugar, etc

- Not to provide children with a sipping cup for drinking

- Until the age of 1 year they must avoid the use of food supplements like infant food, and water other than breast milk

Biswas S | Volume 2; Issue 2 (2021) | JDHOR-2(2)-032 | Short Communication

Citation: Kashyap N, et al. Pediatric Dentistry: Challenges during COVID-19 Pandemic. J Dental Health Oral Res. 2021;2(2):1-9. 
- Follow nutrition guidelines according to their appropriate age and stop bottle feeding

According to the caries risk assessment patients can be classified and treated according to their caries threat level. Preventive care can be given by 1000 ppm fluoridated toothpastes, by topical application of fluoride varnishes at specific time interval and by prescribing additional remineralizing agents for arresting/reversing white spot lesions [9].

\section{Post-Operative Period}

The postoperative period involves the patient's care and supervision after dental procedures. Parents are instructed to be more vigilant in any changes in children after treatment. And in case observed any signs and symptoms to be informed immediately. As well as implementing teledentistry and triage protocols which provides a pragmatic approach to evaluate and record the oral health status postoperatively and hence improve the overall delivery of oral care [10].

Adaptation of techniques and equipments

\begin{tabular}{|l|l|}
\hline \multicolumn{1}{|c|}{ Affected patients } & \multicolumn{1}{c|}{ Adaptation of techniques and equipments } \\
\hline All children & $\begin{array}{l}\text { - The air-water syringe must be used with care; and replace washing with a syringe with } \\
\text { saline. } \\
\text { - Preference for drying with cotton and gauze. } \\
\text { - Minimize the use of the dental spittoon, with constant saliva suction, preferably with } \\
\text { dental vacuum pump. } \\
\text {-Attention and care in the handling medications and dental materials to avoid cross- } \\
\text { infection. }\end{array}$ \\
\hline $\begin{array}{l}\text { Children who require } \\
\text { protective stabilization }\end{array}$ & $\begin{array}{l}\text { The companion must assist in patient restraint with mask and goggles. The assistance } \\
\text { aims not to overload the dental staff, since contact with body fluids such as sweat is } \\
\text { inherent to this type of approach. }\end{array}$ \\
\hline $\begin{array}{l}\text { Immunologically } \\
\text { compromised children }\end{array}$ & $\begin{array}{l}\text { They should be scheduled as the first appointment of the day, minimizing the risk of } \\
\text { exposure to the environment, with increased attention to infection control and air } \\
\text { turnover, given their increased susceptibility when infected by SARS-CoV-2, in addition } \\
\text { to having medical contact for clarifications }\end{array}$ \\
\hline $\begin{array}{l}\text { Children who require } \\
\text { urgent treatments }\end{array}$ & $\begin{array}{l}\text { Such children should have their procedures maximized, preferably aimed at the adequacy } \\
\text { of the oral environment, and avoiding recurring visits to the dental office. }\end{array}$ \\
\hline $\begin{array}{l}\text { Children with caries } \\
\text { lesions with or without } \\
\text { restorative needs }\end{array}$ & $\begin{array}{l}\text { Consider the risk of dental caries, directing strategies for the treatment of active caries } \\
\text { lesions in enamel and dentin; Use fluoride varnishes, silver diamine fluoride, pit and } \\
\text { fissure sealants, temporary and atraumatic restorations, in addition to constant } \\
\text { Reinforcement of the diet and use of fluoride toothpaste. Restorative treatment must be } \\
\text { performed by quadrants under rubber dam isolation, reducing the production of saliva, } \\
\text { aerosol and particles Contaminated by blood. If possible, choose non-aerosol-generating } \\
\text { restorative procedures. Use high-speed handpiece without water spray; and give } \\
\text { preference to manual instruments and low rotation }\end{array}$ \\
\hline
\end{tabular}

Figure 1: Adaptation of techniques and equipments. 


\section{COVID-19 Vaccination among the Pediatric Population}

As evident from many studies children are not considered as super carriers of COVID-19 infections. They show milder symptoms as compared to adult population. Of the total COVID19 cases in the United States of America 14.1\% comprised of children. Canada became the first country to begin Vaccination among children of 12-15 years of age on $5^{\text {th }}$ May, 2021. According to the recent update from World Health Organisation published on $14^{\text {th }}$ July 2021 unless, the children are at a high risk of COVID-19 infection, it is less urgent to vaccinate them than the adult population. However, WHO's Strategic Advisory Group of Experts (SAGE) has concluded that the Pfizer/BioNTech vaccine is suitable for use by people aged 12 years and above. Children aged between 12 and 15 who are at high risk may be offered this vaccine alongside other priority groups for vaccination [11]. The U.S. Food and Drug Administration expanded the Emergency Use Authorization (EUA) for the Pfizer-BioNTech COVID-19 Vaccine for the prevention of coronavirus disease 2019 to include adolescents 12 through 15 years of age. Authorisation of vaccination for the younger population will help us to fight with the immense burden of the public health in the ongoing public. When the adults are getting immune protection through vaccination we cannot neglect the fact that unvaccinated children may become shelter for the virus from epidemiological point of view $[12,13]$.

\section{Conclusion}

A pediatric dentist is the prime oral healthcare provider for a child, so he/she must be well informed about the disease and its threat and treat patients with maximum precaution during these tough situation of COVID-19 pandemic. There is low prevalence of COVID-19 infection among children; however, the actual numbers are likely to be under-reported. Preventive treatment should be provided according to the requirement of the patient to avoid multiple appointments. It is necessary to re-evaluate the procedures done by pediatric dentists taking into account the challenges in terms of disease contagion. Remote education/ teledentistry with parents must be considered as preventive measures for oral health, and for home management of milder oral pathologies for which direct appointment and intervention by a pediatric dentist is not necessary or can be postponed. However in the cases of dental emergencies where immediate intervention is required, strict protection protocols of the subjects involved and surrounding disinfection becomes crucial and of utmost importance in order to minimize the risk of cross infection and contamination. Thus, pediatric dentists are urged to update themselves with recent international and local institutional guidelines. Any practice modifications should be comprehended with professional clinical judgment. 


\section{Conflict of Interest}

There is no conflict of interest following this study.

\section{References}

1. Giovanni Ponti, Monia Maccaferri, Cristel Ruini, Aldo Tomasi, Tomris Ozben. Biomarkers associated with COVID-19 disease progression. Crit Rev Clin Laborat Sci. 2020;57:6,389-99.

2. Marco Ciotti, Massimo Ciccozzi, Alessandro Terrinoni, Wen-Can Jiang, Cheng-Bin Wang, Sergio Bernardini. The COVID-19 pandemic. Crit Rev Clin Laborat Sci. 2020;57(6):365-88.

3. Assaker R, Colas AE, Julien-Marsollier F, Bruneau B, Marsac L, Greff B, et al. Presenting symptoms of COVID-19 in children: a meta-analysis of published studies. Br J Anaesthesia. 2020;125(3):e330-2.

4. Pra Mitra P, Misra S, Sharma P. COVID-19 pandemic in India: what lies ahead. Ind J Clin Biochem. 2020;35(3):257-9.

5. Ludvigsson JF. Children are unlikely to be the main drivers of the COVID-19 pandemic- A systematic review. Acta Paediatr. 2020;109:1525-30.

6. Centers for disease control and prevention. Interim Infection Prevention and Control Guidance for Dental Settings during the Coronavirus Disease 2019 (COVID-19) Pandemic. 2020.

7. Xu Y, Li X, Zhu B, Liang H, Fang C, Gong Y, et al. Characteristics of pediatric SARS CoV-2 infection and potential evidence for persistent fecal viral shedding. Nat Med. 2020;26(4):502-5.

8. Luzzi V, Ierardo G, Bossù M, Polimeni A. COVID-19: Pediatric Oral Health during and after the Pandemics. Appl Sci. 2020;10:1-8.

9. Rathore V, Galhotra A, Pal R, Sahu KK. COVID-19 pandemic and children: a review. J Pediat Pharmacol Therape. 2020;25(7):574-85.

10. Luo L, Liu D, Liao X, Wu XB, Jing QL, Zheng JZ, et al. Modes of contact and risk of transmission in COVID19 among close contacts. MedRxiv. 2020.

11. World Health Organisation, COVID-19 advice for public: Getting Vaccinated. July 14, 2021.

12. HOD D, Mahendra Azad, Preetham Ravuri, Sirisha Kommuri, Heena Tiwari. The challenging perception of pediatric dental practice post COVID the new normal. 2020.

13. Ismail AF. Managing pediatric dental patients during the SARS-CoV-2 pandemic. J Int Oral Health. 2020;12(8):S80-4. 